\title{
The Use of New Mobile and Gaming Technologies for the Assessment and Rehabilitation of People with Ataxia: a Systematic Review and Meta-analysis
}

\author{
Eleonora Lacorte $^{1} \cdot$ Guido Bellomo $^{1} \cdot$ Sara Nuovo $^{2} \cdot$ Massimo Corbo $^{3} \cdot$ Nicola Vanacore $^{1} \cdot$ Paola Piscopo $^{4}$
}

Accepted: 1 November 2020 / Published online: 14 November 2020

(C) The Author(s) 2020

\begin{abstract}
There are no currently available disease-modifying pharmacological treatments for most of the chronic hereditary ataxias; thus, effective rehabilitative strategies are crucial to help improve symptoms and therefore the quality of life. We propose to gather all available evidence on the use of video games, exergames, and apps for tablet and smartphone for the rehabilitation, diagnosis, and assessment of people with ataxias. Relevant literature published up to June 8, 2020, was retrieved searching the databases PubMed, ISI Web of Science, and the Cochrane Database. Data were extracted using a standardized form, and their methodological quality was assessed using RoB and QUADAS-2. Six studies of 434 retrieved articles met the predefined inclusion/exclusion criteria. Two of them were diagnostic, while 4 were experimental studies. Studies included participants ranging from 9 to 28 in trials and 70 to 248 in diagnostic studies. Although we found a small number of trials and of low methodological quality, all of them reported an improvement of motor outcomes and quality of life as measured by specific scales, including the SARA, BBS, DHI, and SF-36 scores. The main reason for such low quality in trials was that most of them were small and uncontrolled, thus non-randomized and unblinded. As video games, exergames, serious games, and apps were proven to be safe, feasible, and at least as effective as traditional rehabilitation, further and more high-quality studies should be carried out on the use of these promising technologies in people with different types of ataxia.
\end{abstract}

Keywords Video games $\cdot$ Mobile applications $\cdot$ Technology $\cdot$ Rehabilitation $\cdot$ Ataxia $\cdot$ Systematic review

\section{Background}

Ataxia, or lack of voluntary coordination of muscle movements, has an overall prevalence of around 26 per 100,000 in European pediatric population [1], 8.4 per 100,000 (95\% CI 7.2 to 11.6) for idiopathic late-onset cerebellar ataxia (LOCA), and 1.8 per 100,000 (95\% CI 0.8 to 2.7) for inherited

Paola Piscopo

paola.piscopo@iss.it

1 National Centre for Disease Prevention and Health Promotion, Italian National Institute of Health, Rome, Italy

2 Department of Human Neuroscience, Sapienza University of Rome, Rome, Italy

3 Department of Neurorehabilitation Sciences, Casa Cura Policlinico (CCP), Milan, Italy

4 Department of Neuroscience, Italian National Institute of Health, Viale Regina Elena, 00161 Rome, Italy
LOCA [2]. Based on the population living in Europe, we can estimate about 525,000 cases in all ages (1300 in pediatric population and 431,200 and 92,500 for idiopathic and inherited LOCA, respectively) (https://ec.europa.eu/eurostat). Ataxia is a common neurological sign that might be due to several different neurological conditions, including brain tumors, brain injuries, stroke, infections (e.g., varicella), toxicity, or genetic causes. Its evolution can be acute, subacute, episodic, or chronic, with the latter including both progressive and non-progressive forms. The diagnosis of the underlying cause is a crucial step, and might be a long process in case of chronic or episodic ataxias, due to the rarity and complexity of these conditions [3]. Cerebellar ataxia is typically among the core features of these diseases, affecting motor skills, eye movements, balance, and coordination, thus significantly affecting activities of daily living (ADL). In some hereditary conditions, ataxia is part of a multisystem dysfunction, which may cause intellectual disability and/or other neurological symptoms, spine deformities such as 
scoliosis, and/or several other disorders affecting different organs (e.g., cardiomyopathy, congenital cataracts, optic nerve atrophy, retinal dystrophy, and deafness) [4-8].

There are no currently available disease-modifying pharmacological treatments for most of the chronic hereditary ataxias, thus effective rehabilitative strategies are crucial to improve symptoms and therefore the quality of life [9].

Rehabilitative and physiotherapeutic interventions are increasingly being tested in clinical studies for the treatment of people with different ataxias. Previous studies showed that treatments based on demanding gait and balance tasks can increase postural stability and lead to reduce the dependency of patients on walking aids, thus increasing their independence in ADL [10-13].

Non-ambulant patients with more severe ataxias were also shown to benefit from locomotion and treadmill training with or without body weight support [9].

In this framework, some recent studies have shown that the use of some virtual reality tools can be effective for the treatment of people with progressive ataxias [14]. Virtual reality is often incorporated within exergames, which are video games designed as easier, more entertaining, and more appealing exercise tools compared to usual, traditional, training systems. Exergaming is defined as technology-driven physical activities, such as video game play, that requires participants to be physically active or exercise in order to play the game [15]. Exergames usually include coordination and balance tasks and are specifically structured to enhance the participation and adherence of the player/participant to the game/intervention. As rehabilitation for people with ataxia can be challenging, these games could be an extremely powerful and useful tool to improve rehabilitative interventions in these patients [16]. As exergames are usually extremely enjoyable and easy to play, do not require the constant presence of a trainer to monitor the activity, and are usually easily available and not very expensive, including these type of games in the rehabilitation program could also help moving the whole intervention, or part of it, from a clinical setting to a home setting, and could help to turn a rehabilitation session into more enjoyable activities, particularly in children. Moreover, the use of exergames could help to reach a higher number of patients by minimizing the access to the health system, as patients could be able to follow the whole rehabilitation program, or even part of it, at home.

Exergames have been proven as effective in improving motor and cognitive symptoms in several neurological and neurodegenerative diseases $[17,18]$. A relatively recent systematic review [19] concluded that consistent evidence supports the effectiveness of rehabilitation in improving motor function, ataxic symptoms, and balance in patients with chronic ataxias, including rehabilitation using serious games and exergames. Furthermore, the therapeutic use of commercially available exergame systems and applications for smartphones and tablets can be very cost-effective and improve quality of life and social inclusion, minimizing the perception of disability. However, no systematic reviews nor meta-analyses are currently available on the effectiveness of this kind of technology specifically in patients with chronic ataxias. For all these reasons, we deemed it relevant to review all existing evidence on the use of this technology in patients with ataxias. Therefore, the objective of this review was to gather, qualitatively assess, and summarize the results, both narratively and through a meta-analysis, from all available evidence on the use of video games, exergames, and apps for tablet and smartphone for the rehabilitation, diagnosis, and assessment of people with ataxias.

\section{Methods}

This systematic review was carried out according to the methodology reported in the Cochrane Handbook for Systematic Reviews [20] and followed the PRISMA statement for reporting systematic reviews and meta-analyses [21, 22]. A structured bibliographic search was performed on the databases PubMed, ISI Web of Science, and the Cochrane Database of Systematic Reviews using the following search terms: ("app" OR "apps" OR "smartphone" OR "smartphones" OR "smart-phone" OR "smart-phones" OR "smart phone" OR "smart phones" OR "tablet"” OR "mobile" OR "game" OR "kinect" OR "nintendo" OR "games" OR "gaming" OR exergam* OR "virtual reality" OR "augmented reality") AND (*ataxi* OR "joubert*"). No limitations were applied for date of publication, study design, nor language. The bibliographic references of selected studies were also browsed to identify further possibly relevant literature. Two independent reviewers (EL, PP) initially selected studies based on their pertinence with and relevance to the topic of the review. Disagreements were solved by discussion or by a third independent reviewer (NV). Relevant literature published up to June 8, 2020, was retrieved searching the databases. Selected studies were retrieved in full text, and the following predefined inclusion/exclusion criteria were applied. We only included (1) experimental and/or observational studies; (2) studies that reported data on the use of technologies such as computer games, gaming consoles, tablet or smartphone apps, and/or devices for augmented or virtual reality for the rehabilitation and/or treatment of people with ataxia; (3) studies that enrolled people with ataxia of any age class; (4) studies that enrolled patients with any type of ataxia; and (5) studies that reported enough information and data to allow for an adequate quality assessment and a summary of evidence. We excluded (1) conference proceedings, letters, abstracts, editorials, narrative reviews, systematic reviews, meta-analyses, case-reports, or case-series; and (2) studies reporting only narrative or non-quantifiable results. 
Systematic reviews were not included, but were anyway selected and analyzed to search their bibliographies, and to check for consistency of results.

The methodological quality of all included studies was assessed by 3 independent reviewers (PP, EL, GB) using The Cochrane Collaboration's tool for assessing risk of bias (RoB)in randomized trials [23], and the QUADAS-2 tool for diagnostic studies [24]. The RoB tool includes seven specific domains aimed at assessing selection bias, performance bias, detection bias, attrition bias, reporting bias, and other potential sources of bias, and requires that enough details are provided to adequately assess the risk of bias, which is defined as "low risk," "high risk," or "unclear risk." The QUADAS-2 tool includes 4 domains analyzing the adequateness of the methodology adopted for patient selection, the choice and management of the index test and the reference standard, and the flow and timing with which the tests were administered. It includes also an assessment of concerns about applicability for 3 of the considered domains (i.e., patient selection, index test, reference standard). The tool does not allow for a global scoring, but it provides an overall rating of high, unclear, or low risk of bias for each domain, and an overall rating of high, unclear, or low concern for applicability for the 3 considered domains. Further potential bias or methodological flaws were also addressed. As neither of the tools provides a method to calculate an overall quality score, we calculated it for both scales. For the RoB tool, the overall score was calculated by summing the number of items scored as "low risk of bias," thus having an overall score ranging from 0 to 7 , with higher scores indicating higher quality. For the QUADAS-2 tool, the score was calculated by summing the number of items scored as "low risk of bias" or "low applicability concern," thus obtaining an overall score ranging from 0 to 7 , with higher scores indicating higher quality.

Data extraction was performed by 3 independent reviewers (GB, PP, EL), and data were summarized in specifically designed standardized forms. Disagreements were resolved by discussion between the reviewers.

Due to a high heterogeneity, a meta-analysis was carried out using data from the only 2 experimental studies reporting results for the same outcome measure. The summary tables for the qualitative assessment and the meta-analysis were performed using the software RevMan version 5.3 provided by the Cochrane Collaboration.

\section{Results}

A total of 661 records were retrieved through the bibliographic searches. No articles were retrieved by browsing the references of included studies. No systematic reviews nor metaanalyses were available on this topic. Of the studies retrieved through bibliographic searches, 7 studies were selected based on their relevance and pertinence to the topic of the review. Full texts were gathered and, after applying the predefined inclusion and exclusion criteria, only 6 studies were included in the review. One study was excluded, as it was a letter to the editors reporting preliminary data on 1 patient who was subsequently enrolled in the study published in 2017 by Schatton et al. [25].

The flow diagram of literature is reported in Fig. 1.

\section{Qualitative Assessment of Included Studies}

Four studies were experimental studies, with 1 controlled trial [26] and 3 single-group trials [27-29]. Two studies were categorized as diagnostic studies [30, 31].

All included experimental studies investigated the effectiveness of either available video games for both Nintendo Wii and Microsoft Xbox Kinect [27-29] or specifically developed exergames adopting the Kinect sensor in both children and adults with different ataxias [26].

The 2 diagnostic studies evaluated the accuracy of either an already available serious game coupled with the Kinect sensor [30] or a specifically developed app for tablet and smartphone [31] to assess motor symptoms in patients with ataxias, and to discriminate between healthy subjects and patients with ataxias.

A summary of the results, main characteristics, and quality of included studies is reported in Table 1.

The overall methodological quality of all included studies was medium-low, with 1 trial reaching a score of 4 [26], 2 trials having a score of 3, 1 trial having a score of 1 [29], and both diagnostic studies having a score of 4 .

The main reason for low scores in trials was that 3 studies did not include a control group [27-29]; thus, patients were not randomized nor blinded. All trials included small samples (ranging from 9 to 28 participants), and the only randomized study [26] applied a block randomization in 9 subjects, thus leading to significant differences (e.g., Walking speed, Ninehole peg test) in the characteristics of subjects between the experimental and the control group. Moreover, 1 trial [29] reported and analyzed data in an unclear way, leading to a high risk of incomplete and selective reporting (e.g., means and ICs are not reported). However, ataxias are relatively rare conditions, the technologies tested are difficult to standardize, and it is relatively difficult to blind participants to them.

The main risk of bias in diagnostic studies was that they were designed as case-control studies, thus the reference standard was carried out before the index test, and raters were not blind to the diagnoses. However, the technology adopted was objective; thus, the risk of interpreting results in an altered way due to the knowledge of the diagnosis is relatively low. Both trials enrolled small samples of selected patients (e.g., from a consortium), and one study enrolled subjects with different diagnoses. As previously stated, ataxias are relatively 
Fig. 1 Flow diagram of the literature

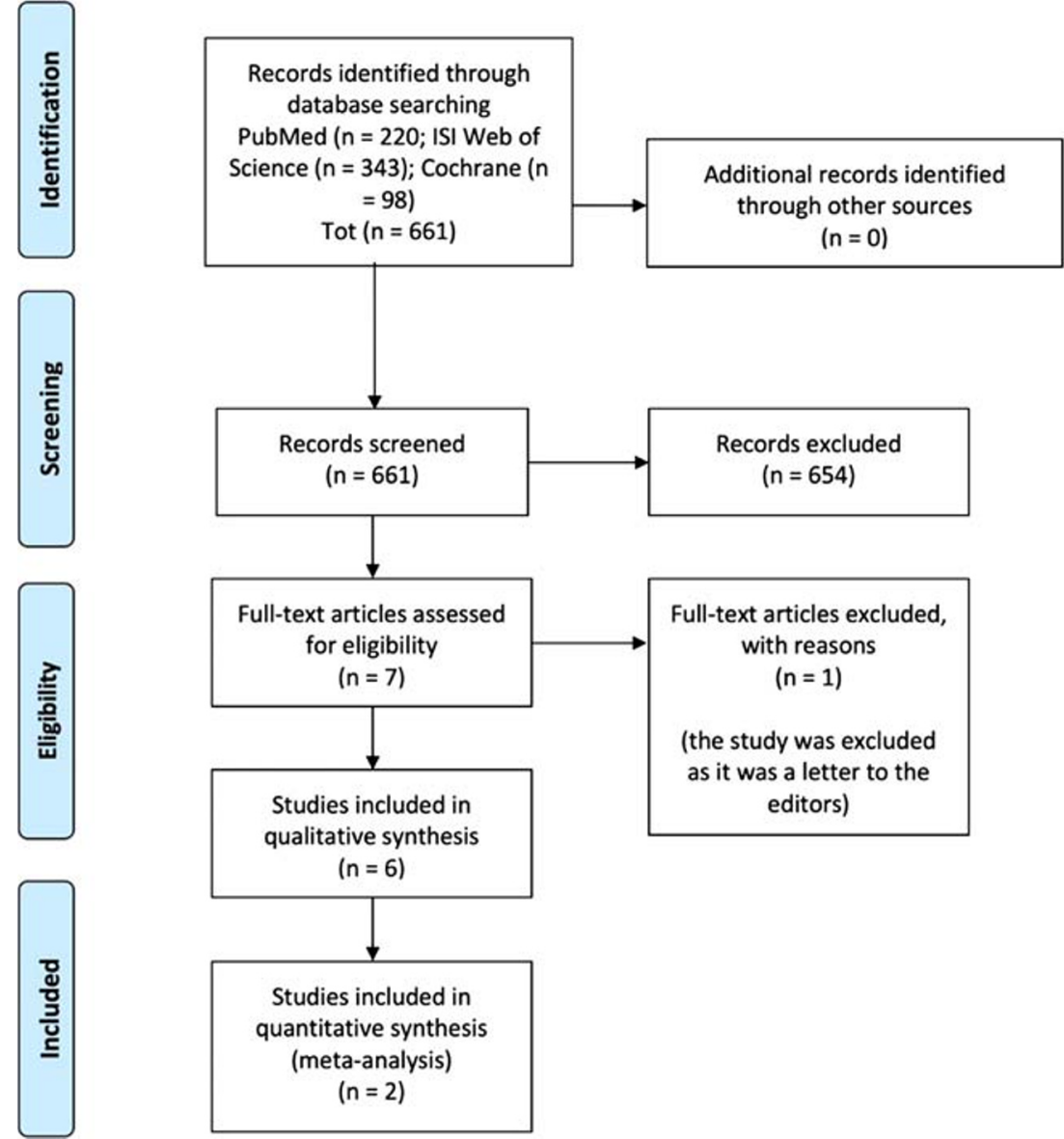

rare diseases, thus enrolling a large number of consecutive or random subjects might be very difficult.

A summary of the qualitative assessment of all included studies is reported in Fig. 2 for diagnostic studies and Fig. 3 for trials.

As for the included trials, a high heterogeneity was observed in the characteristics of the populations enrolled, in the type of interventions used, and in the outcomes considered.

Three studies included participants aged $<18$ years, while 1 included only adult subjects. Age ranges were extremely heterogeneous, with 1 study enrolling subjects aged 11-20 [28], 1 enrolling subjects aged 15 to 70 [29], 1 including subjects aged 6-29 [27], and 1 including adults aged 51-60 [26].

All 4 trials used exergaming for the rehabilitation of both adults and children with different types of ataxia, and all employed commercially available consoles and video games. Specifically, 1 study used the Nintendo Wii [29], 1 used the Xbox Kinect [28], 1 used both the Nintendo Wii and the Xbox Kinect [27], and 1 used the Kinect sensor along with a specific exergame program [26].

Only 1 study included a control group treated with conventional training [26]; all the remaining studies were based on "inter-subject" control, meaning that they compared performances pre- and post-training.

Three trials used the SARA score as their primary outcome measure, while 1 used the Dizziness Handicap Inventory (DHI), the Berg Balance Scale (BBS), and the Short-Form 36-Items (SF-36) scores as outcome measures [29]. Considering the relative homogeneity of the outcome measure in three trials, we attempted a meta-analysis of the mean change from baseline to post-training in the SARA score. However, 1 trial [26] did not report the mean scores and SD pre- and post-training; thus, we excluded it from the metaanalysis. Means and SDs of the remaining 2 trials [27, 28] were calculated using the individual patient data reported in the studies, and for 1 of the trials, post-test scores [28] were extracted from the provided graph (Fig. 4).

\section{Description of Included Studies}

The study by Wang et al., the only controlled study [26], was a pilot trial enrolling 9 subjects with spinocerebellar ataxia (SCA) type 3 aged 51 to 60 years (mean age 54) randomized to either exergaming with Kinect sensor or to conventional balance and coordination training. The experimental group $(n=5)$ followed 3 sessions of 40-min per 


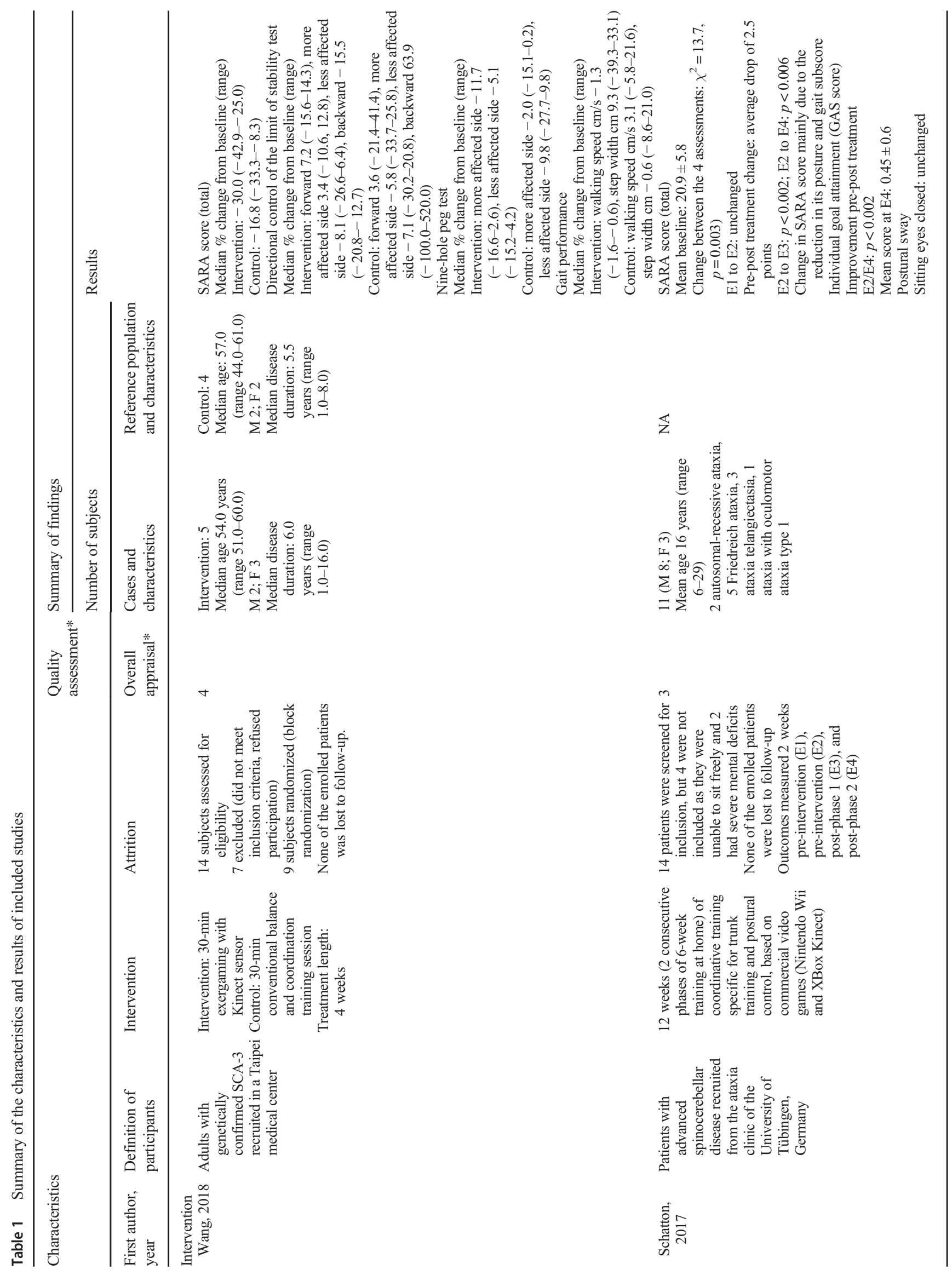




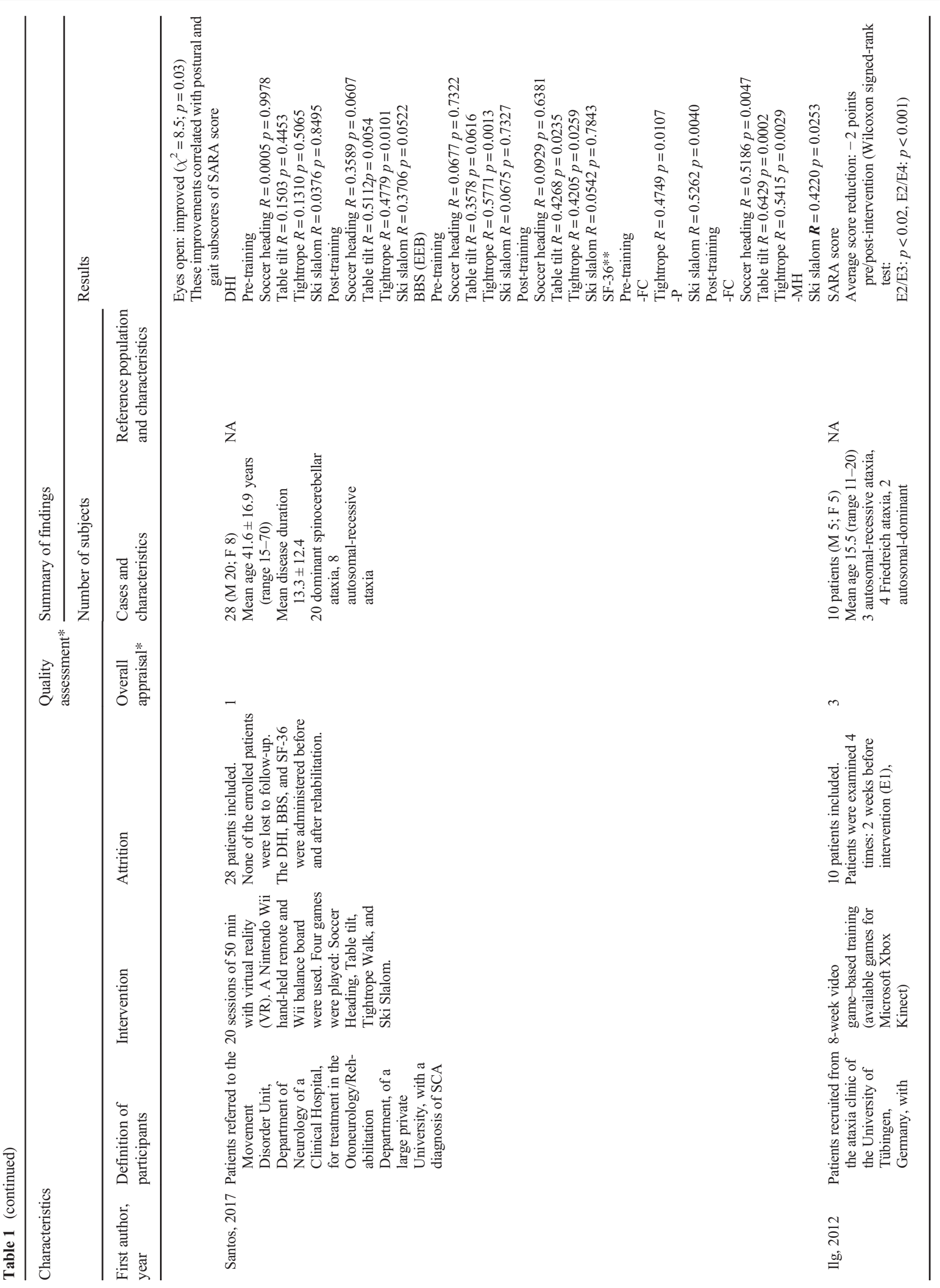




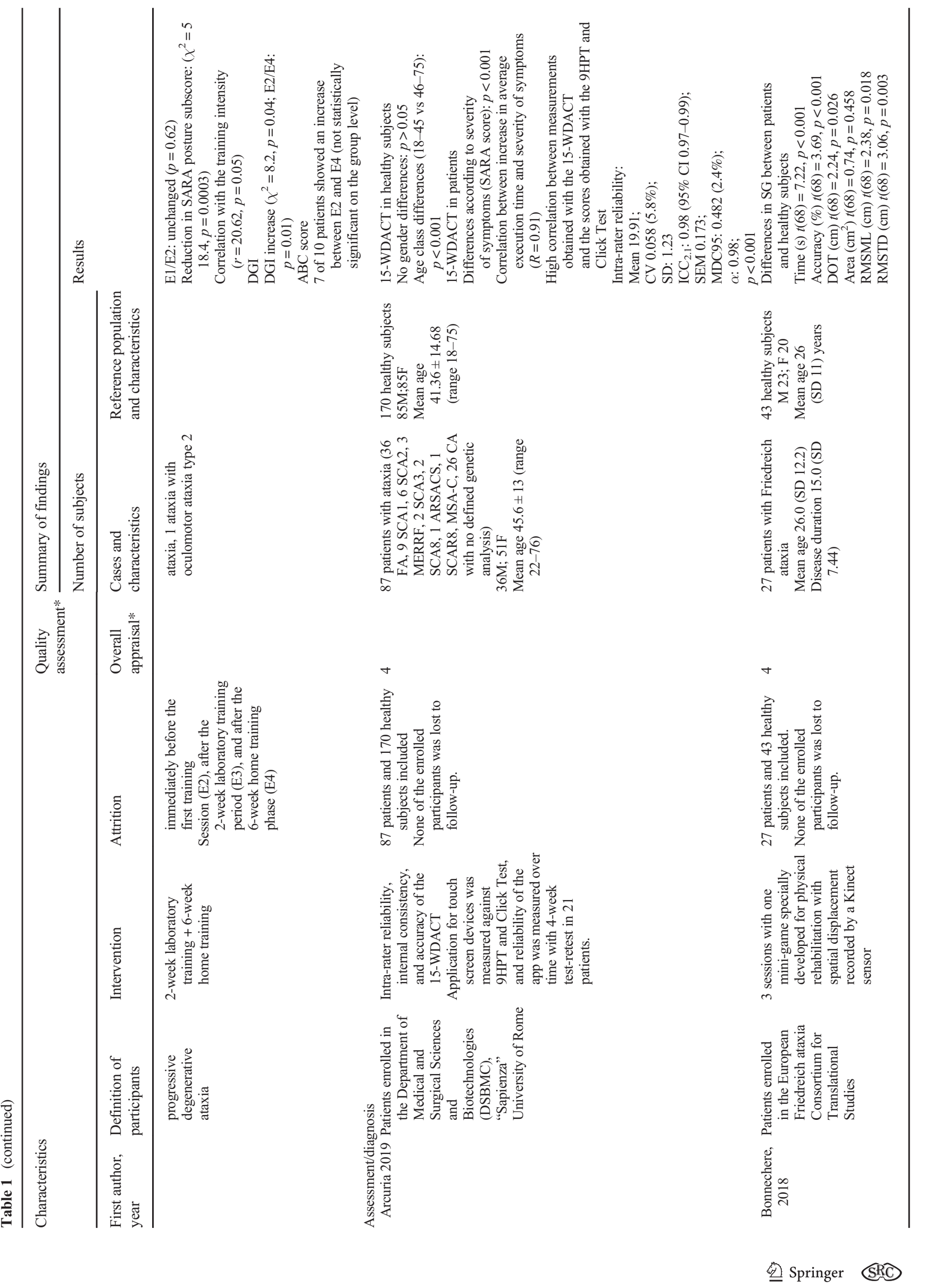




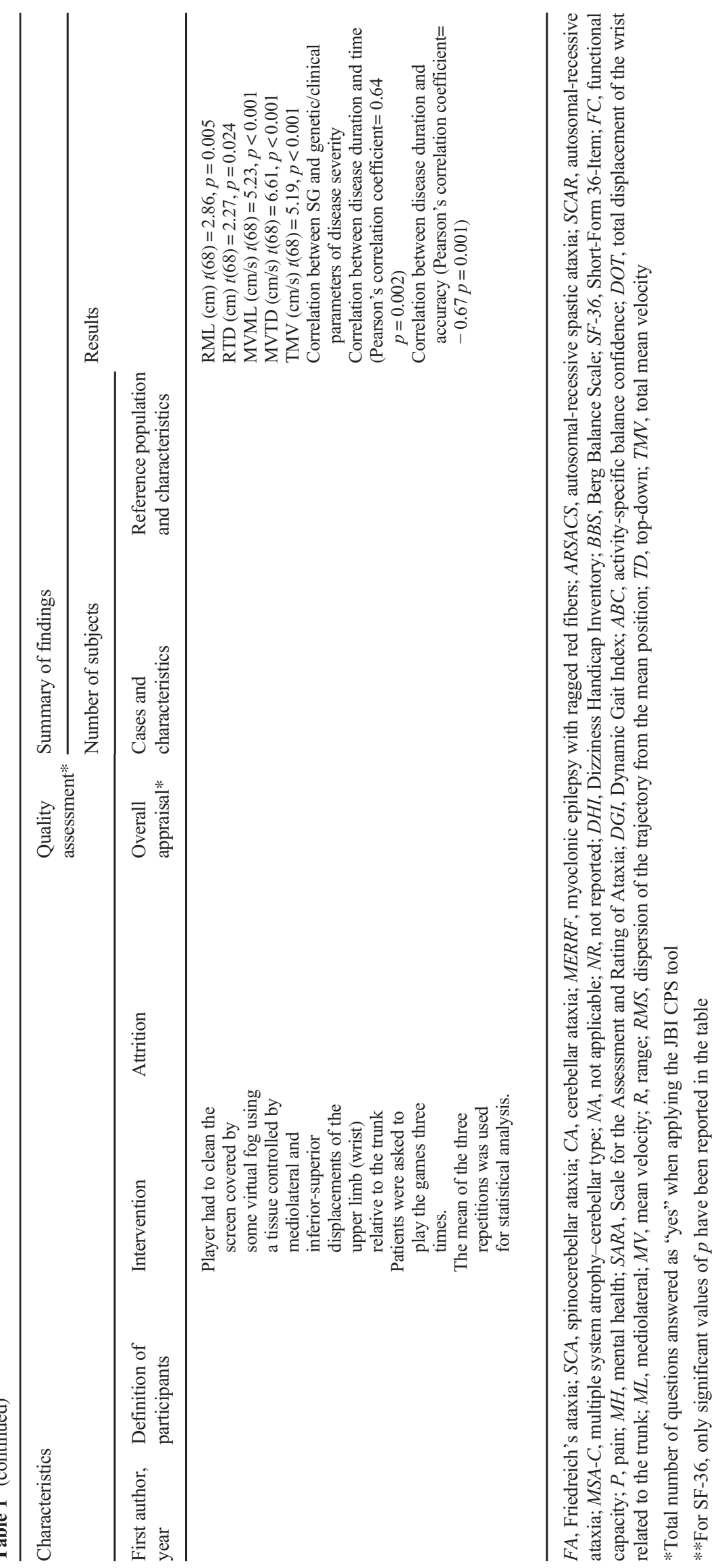

SRC 算 Springer 
a

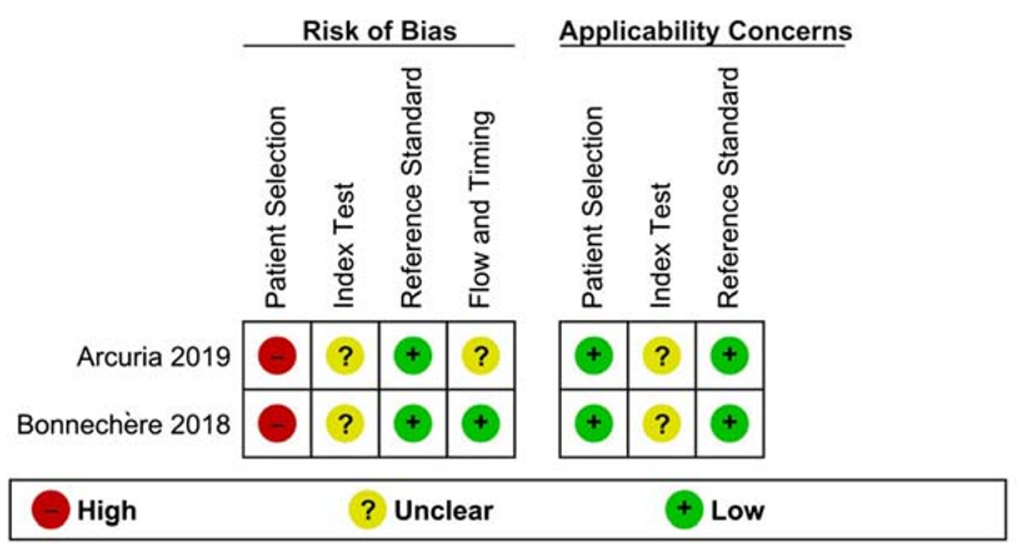

b

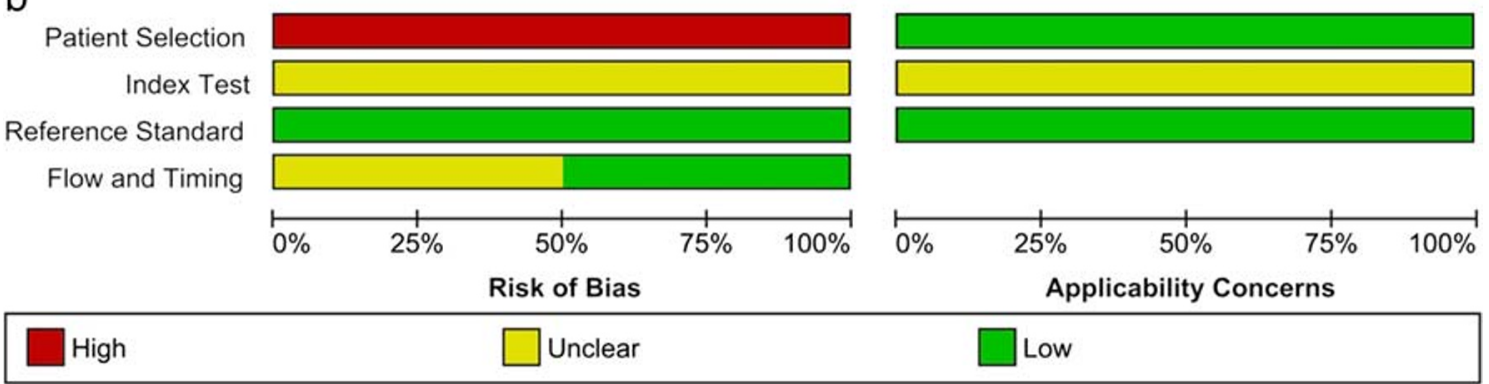

Fig. 2 Results from the risk of bias assessment of included diagnostic studies. a Summary table of the assessment for each item for each study. b Graph plotting the distribution of assessments across studies for each item

week for 4 weeks of an exergaming program intervention (a specifically designed game including a reaching task, a pointing task, a following task, and an avoiding task) using a Kinect sensor. Results showed a median decrease in the SARA score of $-30.0 \%$ (range $-42.9--25.0$ ) in the exergaming group and $-16.8 \%(-33.3--8.3)$ in the control group, with a reduction up to $-50 \%$ of the gait-posture subscore in the exergaming group.

Among non-controlled studies, the trial by Santos et al., the largest one [29], which was also the one with the lowest quality score, enrolled 28 subjects with undefined SCA $(n=7)$, $\operatorname{SCA} 3(n=5), \operatorname{SCA} 10(n=5), \operatorname{SCA} 2(n=2), \operatorname{SCA} 4(n=1)$, and autosomal-recessive cerebellar ataxia (ARCA) $(n=8)$. All subjects underwent a therapeutic body-balance training for vestibular rehabilitation using the Nintendo Wii handheld remote and Wii balance board with 4 commercially available balance games (Soccer heading, Table tilt, Tightrope walk, Ski slalom). Results report an improvement in balance, measured with the Berg Balance Scale (BBS), and in dizziness, measured with the Dizziness Handicap Inventory (DHI). However, data are not clearly reported in the publication and crude values pre- and post-training for each outcome measure are not detailed.

The study by Schatton et al. [27], another non-controlled trial, enrolled 11 subjects diagnosed with Friedreich ataxia (FA) $(n=5)$, ataxia telangiectasia $(n=3)$, autosomalrecessive ataxia with no identified genetic cause $(n=2)$, or ataxia with oculomotor ataxia type $1(n=1)$. All participants underwent 12 weeks of coordinative training with Nintendo Wii and Xbox Kinect using commercially available video games. The study used the SARA score as the main outcome measure, and reported a significant short-term (at 6 weeks; $p<0.002$ ) and long-term (at 12 weeks; $p<0.006$ ) improvement in the overall SARA score from baseline to post-training, mainly driven by improvements in posture and gait.

The study by Ilg et al., the smallest of the non-controlled trials [28], enrolled 10 patients with FA $(n=4)$, ataxia with oculomotor ataxia type $1(n=1)$, and autosomal-recessive $(n=3)$ and autosomal-dominant $(n=2)$ ataxia without genetic diagnosis. All patients underwent an 8-week video gamebased coordination training using 3 commercially available Microsoft Xbox Kinect games (Table tennis; Light race; 20,000 Leaks). The study used the SARA score as the primary outcome measure, with results showing a significant shortterm (at 2 weeks; $p<0.02$ ) and long-term (at 8 weeks; $p<0.001)$ improvement in the overall score from baseline to post-training, mainly due to an improvement in posture $(p=$ $0.0003)$. Results also showed a significant improvement in the overall dynamic balance $(p=0.01)$.

As for diagnostic studies, the study by Arcuria et al., the largest one [31], enrolled 87 patients affected by FA $(n=36)$, SCA1 $(n=9)$, SCA2 $(n=6)$, myoclonic epilepsy with ragged red fibers (MERRF) $(n=3), \operatorname{SCA} 3(n=2), \operatorname{SCA} 8(n=2)$, autosomal-recessive spastic ataxia of Charlevoix-Saguenay 
Fig. 3 Results from the risk of bias assessment of included RCTs. a Summary table of the assessment for each item for each study. b Graph plotting the distribution of assessments across studies for each item a

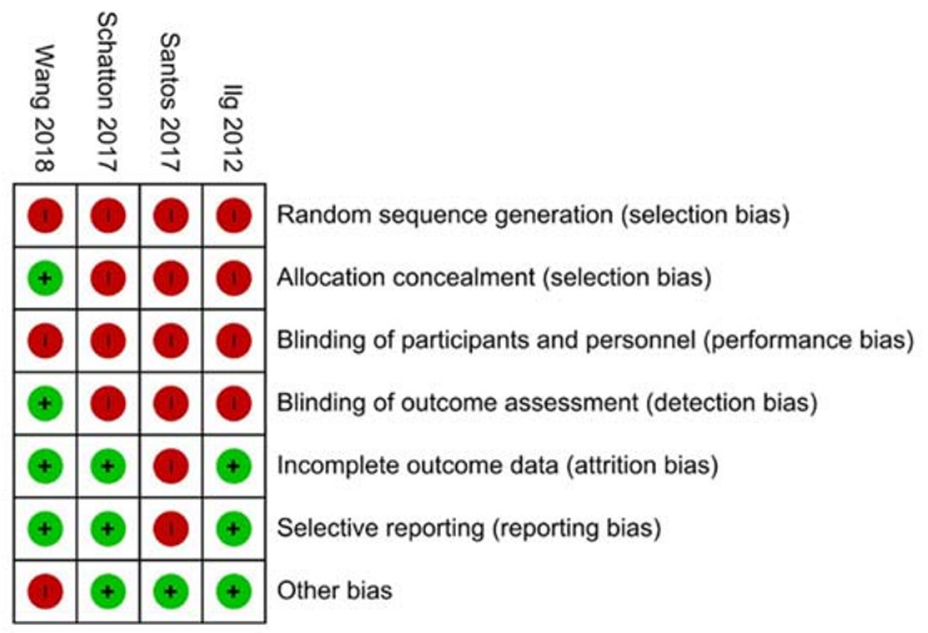

b

Random sequence generation (selection bias)

Allocation concealment (selection bias)

Blinding of participants and personnel (performance bias)

Blinding of outcome assessment (detection bias)

Incomplete outcome data (attrition bias)

Selective reporting (reporting bias)

Other bias

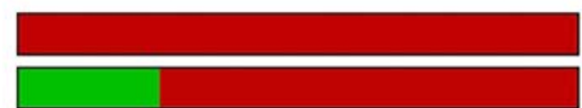

\section{.}

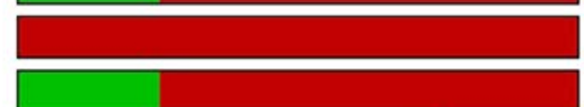

\section{$\square$}
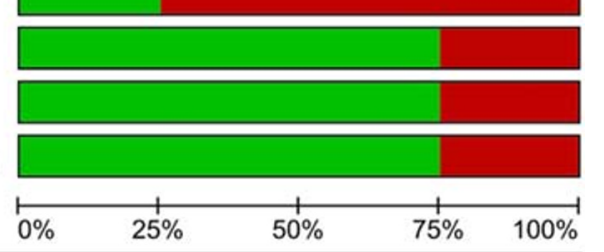

Low risk of bias

Unclear risk of bias
(ARSACS) $(n=1)$, autosomal-recessive ataxia type 8 (SCAR8) $(n=1)$, multiple system atrophy-cerebellar type (MSA-C) $(n=1)$, or CA with no defined genetic diagnosis $(n=26)$, as well as 170 sex- and age-matched healthy subjects. All participants were tested with the 15-White Dots APP-Coo-Test (15-WDACT), an application specifically developed for tablets-PC, as index test, and the Nine-hole peg test (9HPT) and Click Test as reference standards. In healthy subjects, results showed no significant differences based on gender, while a significant difference in the mean execution time between the 18-45 age class and the 46-75 age class was reported $(p<0.001)$. In patients, 15-WDACT execution time increased along with disease severity $(R=0.91)$, and a high correlation was observed between measurements obtained with the 15-WDACT and the scores obtained with the 9HPT and Click Test. Moreover, results showed a high intra-rater reliability, accuracy, and internal consistency.

The study by Bonnechere et al., the smaller study classified as diagnostic [30], enrolled 27 patients with FA and 43 healthy subjects. All subjects participated in 3 sessions of a specifically designed mini-game (Wipe Out game), with spatial displacement recorded by a Kinect sensor. Highly significant differences were observed for time and accuracy of execution between patients and controls (both $p<0.001$ ). Among patients, significant correlations were observed between age and time of execution $(p=0.015)$ and accuracy

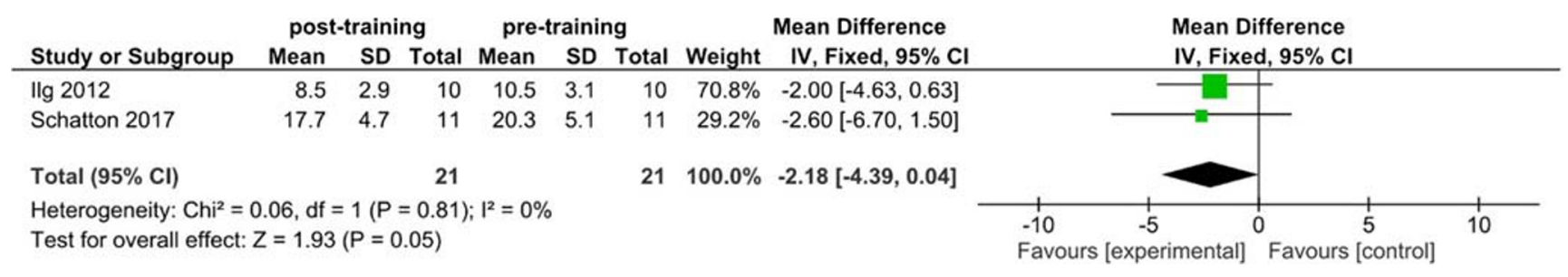

Fig. 4 Results from the meta-analysis of the subgroup of homogeneous studies 
$(p=0.004)$, as well as between age at diagnosis and speedrelated parameters $(p=0.021)$. A significant correlation was also observed between the Nine-hole peg test and the total displacement of the upper limbs $(p=0.012)$, the area covered $(p=0.025)$, the amplitudes of movements (mediolateral displacement $p=0.041$; top-down displacement $p=0.033$ ). Moreover, a statistically significant correlation was found between disease duration and decreased speed $(p=0.002)$, which was associated with a significant reduction in accuracy $(p=0.001)$.

\section{Discussion}

In this review, we assessed currently available evidence on the use of new mobile and gaming technologies in the assessment and rehabilitation of people with chronic ataxias. Six studies were included in the systematic review, enrolling a number of participants ranging from 9 to 28 in trials and from 70 to 248 in diagnostic studies. The wide difference in the sample sizes between the 2 types of study might be due to the fact that treating a patient within a structured rehabilitative intervention is extremely more challenging in terms of resources than assessing a motor feature. Two of the included studies were diagnostic, while 4 were experimental studies. Although we found a small number of trials and despite their low methodological quality, all of them reported an improvement of motor outcomes and quality of life as measured by specific scales, including the SARA, BBS, DHI, and SF-36 scores. The main reason for such low quality in trials was that most of them were small and uncontrolled, thus non-randomized and unblinded. Ataxias are rare diseases, thus enrolling large samples can be extremely challenging. In addition, a group of patients cannot be eligible for rehabilitation protocols based on gaming and virtual or augmented reality, due to the wide range of comorbidities potentially associated with ataxia. Among these, visual loss, hearing disturbances, intellectual disability, and predominant non-ataxia movement disorders (e.g., spasticity, chorea, Parkinsonism) are usually considered exclusion criteria [27-29]. Moreover, managing a rehabilitative study on patients that, though having the same diagnosis may have widely different phenotypic variants and peculiarities, might be very difficult. However, adopting a multicenter approach and involving organizations of ataxic patients could allow enrolling a larger number of participants, increasing the size of subgroups with homogeneous phenotypes.

We found only 2 diagnostic studies investigating the use of these technologies for the assessment of specific motor functions in people with chronic ataxias. Though having an overall low-quality score, they both reported these tools to be useful and reliable. The low quality of these studies was mainly due to their being designed as case-control diagnostic studies and the enrollment of subjects with different diagnoses, disease duration, and degree of severity. The rarity of the disease, however, makes it virtually impossible to design conventional diagnostic studies.

Overall, a wide heterogeneity was observed across included studies in the participants enrolled, the type of technologies applied, and the outcome measures adopted. This prevented a direct comparison and a cumulative analysis of results. Some studies enrolled patients with different types of ataxias, different age classes, and different levels of severity of symptoms. Only 2 studies used the same technologies, with the same outcome measures. As a result, it is difficult to draw general conclusions on the most appropriate approach and the optimal age of intervention for the investigated diseases.

An additional limitation is that all included studies enrolled participants with progressive (degenerative) ataxias, mostly characterized by cerebellar atrophy and a progressively disabling course. None of the studies included patients with nonprogressive forms, which are typically associated with midbrain-hindbrain malformations (mostly cerebellar hypoplasia and/or dysplasia of variable severity, either isolated or associated to other brainstem defects) [32, 33]. Considering the almost stable course of neurological impairment associated with non-progressive ataxias, it is reasonable to expect a greater and more durable effectiveness of gaming-based rehabilitation in this kind of patients. However, further targeted studies are needed to address this issue.

Video games, exergames, serious games, and apps were proven to be safe, feasible in patients with Parkinson's disease, and at least as effective as traditional rehabilitation [34] specifically in improving balance and fatigue [35], and they also appear to be promising tools in the treatment of children with cerebral palsy [36]. Therefore, further, and more highquality, studies should be carried out on the use of these technologies in people with different types of ataxia. The ideal study to investigate the efficacy and/or effectiveness of any type of interventions would be a randomized, controlled trial. To minimize bias, trials should include a large sample of consecutive patients and randomize them to either treatment or placebo/usual care in a blinded fashion. Maintaining the blindness of both participants and the staff administering the treatment, however, can be impossible, in rehabilitative trials; thus, usually the blindness of the personnel assessing the outcomes should be at least guaranteed. Therefore, additional larger, multicenter randomized controlled trials should be carried out with the objective of providing more structured evidence of the potential efficacy and effectiveness of exergaming in the rehabilitation of children and adults with ataxia.

\section{Conclusion}

Results from the included studies were inconclusive, as there was a wide heterogeneity in the considered outcomes and in 
the type of games and technology adopted; thus, results, except for two studies, could not be aggregated nor directly compared. However, games and apps appeared to be promising in improving the motor symptoms and quality of life of patients with ataxias, or in testing specific types of symptoms. Therefore, as video games, exergames, serious games, and apps were proven to be safe, feasible, and at least as effective as traditional rehabilitation tools in patients with neurodegenerative diseases, further and more high-quality studies should be carried out on the use of these promising technologies in people with different types of ataxia.

Author Contributions All authors contributed to the study conception and design. Literature search, data collection, and analysis were performed by Eleonora Lacorte, Guido Bellomo, and Paola Piscopo. The first draft of the manuscript was written by Eleonora Lacorte and Paola Piscopo, and all authors commented on previous versions of the manuscript. All authors read and approved the final manuscript.

Funding Study funding was supported by the Ministry of Health (Ricerca Finalizzata NET-2013-02356160).

\section{Compliance with Ethical Standards}

Conflict of Interest The authors declare that they have no conflict of interest.

Open Access This article is licensed under a Creative Commons Attribution 4.0 International License, which permits use, sharing, adaptation, distribution and reproduction in any medium or format, as long as you give appropriate credit to the original author(s) and the source, provide a link to the Creative Commons licence, and indicate if changes were made. The images or other third party material in this article are included in the article's Creative Commons licence, unless indicated otherwise in a credit line to the material. If material is not included in the article's Creative Commons licence and your intended use is not permitted by statutory regulation or exceeds the permitted use, you will need to obtain permission directly from the copyright holder. To view a copy of this licence, visit http://creativecommons.org/licenses/by/4.0/.

\section{References}

1. Musselman KE, Stoyanov CT, Marasigan R, Jenkins ME, Konczak J, Morton SM, et al. Prevalence of ataxia in children: a systematic review. Neurology. 2014;82(1):80-9.

2. Muzaimi MB, Thomas J, Palmer-Smith S, Rosser L, Harper PS, Wiles CM, D Ravine, N P Robertson. Population based study of late onset cerebellar ataxia in South East Wales. J Neurol Neurosurg Psychiatry 2004;75(8):1129-1134.

3. Daker-White G, Ealing J, Greenfield J, Kingston H, Sanders C, Payne K. Trouble with ataxia: a longitudinal qualitative study of the diagnosis and medical management of a group of rare, progressive neurological conditions. SAGE Open Med. 2013;1: 2050312113505560.

4. Romani M, Micalizzi A, Valente EM. Joubert syndrome: congenital cerebellar ataxia with the molar tooth. Lancet Neurol. 2013;12(9):894-905.

5. Akbar U, Ashizawa T. Ataxia. Neurol Clin. 2015;33(1):225-48.
6. Braverman NE, Raymond GV, Rizzo WB, Moser AB, Wilkinson ME, Stone EM, et al. Peroxisome biogenesis disorders in the Zellweger spectrum: an overview of current diagnosis, clinical manifestations, and treatment guidelines. Mol Genet Metab. 2016;117(3):313-21.

7. Chang IJ, He M, Lam CT. Congenital disorders of glycosylation. Ann Transl Med. 2018;6(24):477.

8. Bird TD. Hereditary ataxia overview. 1998 Oct 28 [Updated 2019 Jul 25]. In: Adam MP, Ardinger HH, Pagon RA, et al., editors. GeneReviews ${ }^{\circledR}$ [Internet]. Seattle (WA): University of Washington, Seattle; 1993-2019.

9. Ilg W, Bastian AJ, Boesch S, Burciu RG, Celnik P, Claaßen J, et al. Consensus paper: management of degenerative cerebellar disorders. Cerebellum. 2014;13:248-68.

10. Ilg W, Timmann D. Gait ataxia-specific cerebellar influences and their rehabilitation. Mov Disord. 2013;28(11):1566-75.

11. de Oliveira LAS, Martins CP, Horsczaruk CHR, da Silva DCL, Vasconcellos LF, Lopes AJ, et al. Partial body weight-supported treadmill training in spinocerebellar ataxia. Rehabil Res Pract. 2018;2018:7172686.

12. Fonteyn EM, Heeren A, Engels JJ, Boer JJ, van de Warrenburg BP, Weerdesteyn V. Gait adaptability training improves obstacle avoidance and dynamic stability in patients with cerebellar degeneration. Gait Posture. 2014;40(1):247-51.

13. Ataxia UK. Management of the ataxias-towards best clinical practice third edition, July 2016. Available at: https://www.ataxia.org. uk/Handlers/Download.ashx?IDMF=261e0aa4-5ca0-4b90-9db01ecb6ef8738a. (Last visited: 06/08/2020).

14. Ilg W, Synofzik M, Brötz D, Burkard S, Giese MA, Schöls L. Intensive coordinative training improves motor performance in degenerative cerebellar disease. Neurology. 2009;73:1823-30.

15. American College of Sports Medicine. ACSM Information On... Exergaming. 2013. Available at: http://healthysd.gov/wp-content/ uploads/2014/11/exergaming.pdf

16. Synofzik M, Ilg W. Motor training in degenerative spinocerebellar disease: ataxia-specific improvements by intensive physiotherapy and exergames. Biomed Res Int. 2014;2014:583507.

17. Bonnechère B, Jansen B, Omelina L, Van Sint Jan S. The use of commercial video games in rehabilitation: a systematic review. Int $\mathrm{J}$ Rehabil Res. 2016 Dec;39(4):277-90.

18. Mura G, Carta MG, Sancassiani F, Machado S, Prosperini L. Active exergames to improve cognitive functioning in neurological disabilities: a systematic review and meta-analysis. Eur J Phys Rehabil Med. 2018 Jun;54(3):450-62. https://doi.org/10.23736/ S1973-9087.17.04680-9 Epub 2017 Oct 25.

19. Milne SC, Corben LA, Georgiou-Karistianis N, Delatycki MB, Yiu EM. Rehabilitation for individuals with genetic degenerative ataxia: a systematic review. Neurorehabil Neural Repair. 2017;31(7):60922.

20. Higgins JPT, Green S (editors). Cochrane handbook for systematic reviews of interventions version 5.1.0 [updated March 2011]. The Cochrane Collaboration. (2011a). Available from http://handbook. cochrane.org. (last visited: 08/02/2019).

21. Liberati A, Altman DG, Tetzlaff J, Mulrow C, Gøtzsche PC, Ioannidis JP, et al. The PRISMA statement for reporting systematic reviews and meta-analyses of studies that evaluate healthcare interventions: explanation and elaboration. BMJ. 2009;339:b2700.

22. Moher D, Liberati A, Tetzlaff J, Altman DG, The PRISMA Group. Preferred reporting items for systematic reviews and meta-analyses: the PRISMA statement. PLoS Med. 2009;6(6):e1000097.

23. Higgins JP, Altman DG, Gøtzsche PC, Jüni P, Moher D, Oxman AD, Savovic J, Schulz KF, Weeks L, Sterne JA; Cochrane Bias Methods Group; Cochrane Statistical Methods Group. The Cochrane collaboration's tool for assessing risk of bias in randomised trials. BMJ 2011b;343:d5928. 
24. Whiting PF, Rutjes AWS, Westwood ME, Mallett S, Deeks JJ, Reitsma JB, et al. QUADAS-2 Group. QUADAS-2: a revised tool for the quality assessment of diagnostic accuracy studies. Ann. Intern. Med. 2011;155:529-36.

25. Synofzik M, Schatton C, Giese M, Wolf J, Schöls L, Ilg W. Videogame-based coordinative training can improve advanced. Multisystemic Early-Onset Ataxia J Neurol. 2013;260(10):2656-8.

26. Wang RY, Huang FY, Soong BW, Huang SF, Yang YR. A randomized controlled pilot trial of game-based training in individuals with spinocerebellar ataxia type 3. Sci Rep. 2018;8(1):7816.

27. Schatton C, Synofzik M, Fleszar Z, Giese MA, Schöls L, Ilg W. Individualized exergame training improves postural control in advanced degenerative spinocerebellar ataxia: a rater-blinded, intraindividually controlled trial. Parkinsonism Relat Disord. 2017;39: $80-4$.

28. Ilg W, Schatton C, Schicks J, Giese MA, Schöls L, Synofzik M. Video game-based coordinative training improves ataxia in children with degenerative ataxia. Neurology. 2012;79(20):2056-60.

29. Santos G, Zeigelboim DBS, Severiano M, Teive H, Liberalesso P, Marques J, et al. Feasibility of virtual reality-based balance rehabilitation in adults with spinocerebellar ataxia: a prospective observational study. Hearing, Balance and Communication. 2017;15(4): 244-51.

30. Bonnechère B, Jansen B, Haack, Omelina L, Feipel V, Van Sint Jan $\mathrm{S}$, et al. Automated functional upper limb evaluation of patients with Friedreich ataxia using serious games rehabilitation exercises. J Neuroeng Rehabil. 2018;15(1):87.
31. Arcuria G, Marcotulli C, Galasso C, Pierelli F, Casali C. 15-White Dots APP-Coo-Test: a reliable touch-screen application for assessing upper limb movement impairment in patients with cerebellar ataxias. J Neurol. 2019;266(7):1611-22.

32. Doherty D, Millen KJ, Barkovich AJ. Midbrain and hindbrain malformations: advances in clinical diagnosis, imaging, and genetics. Lancet Neurol. 2013;12(4):381-93.

33. Poretti A, Boltshauser E, Doherty D. Cerebellar hypoplasia: differential diagnosis and diagnostic approach. Am J Med Genet C Semin Med Genet. 2014;166C(2):211-26.

34. Garcia-Agundez A, Folkerts A, Konrad R, Caserman P, Tregel T, Goosses M, et al. Recent advances in rehabilitation for Parkinson's disease with exergames: a systematic review. J Neuroeng Rehabil. 2019;16:17.

35. Ribas CG, Alves da Silva L, Ribas Corrêa M, Ghizone Teive H, Valderramas S. Effectiveness of exergaming in improving functional balance, fatigue and quality of life in Parkinson's disease: a pilot randomized controlled trial. Parkinsonism Relat Disord. 2017;38: 13-8.

36. Warnier N, Lambregts S, Port IV. Effect of virtual reality therapy on balance and walking in children with cerebral palsy: a systematic review. Dev Neurorehabil. 2019:1-17.

Publisher's Note Springer Nature remains neutral with regard to jurisdictional claims in published maps and institutional affiliations. 